\title{
On the behavior of surface electromyographic variables during the menstrual cycle
}

\author{
Fabiano Araujo Soares ${ }^{1,2}$, Sauro Emerick Salomoni ${ }^{1,3}$, \\ Wilson Henrique Veneziano ${ }^{4}$, Joao Luiz Azevedo de Carvalho ${ }^{1}$, \\ Francisco Assis de Oliveira Nascimento ${ }^{1}$, Kenia Fonseca Pires ${ }^{5}$ \\ and Adson Ferreira da Rocha ${ }^{2,5}$ \\ ${ }^{1}$ Department of Electrical Engineering, University of Brasilia, Brasilia-DF, Brazil \\ ${ }^{2}$ UnB-Gama Faculty, University of Brasilia, Gama-DF, Brazil \\ ${ }^{3}$ Center for Sensory-Motor Interaction, Aalborg, Denmark \\ ${ }^{4}$ Department of Computer Science, University of Brasilia, Brasilia-DF, Brazil \\ ${ }^{5}$ Medical Sciences Program, University of Brasilia, Brasilia-DF, Brazil \\ E-mail: fabianosoares@unb.br and adson@unb.br
}

Received 17 October 2010, accepted for publication 2 March 2011 Published 28 March 2011

Online at stacks.iop.org/PM/32/543

\begin{abstract}
The goal of this work is to study the behavior of electromyographic variables during the menstrual cycle. Ten female volunteers $(24.0 \pm 2.8$ years of age $)$ performed fatiguing isometric contractions, and electromyographic signals were measured on the biceps brachii in four phases of the menstrual cycle. Adaptations of classical algorithms were used for the estimation of the root mean square (RMS) value, absolute rectified value (ARV), mean frequency $(\mathrm{MNF})$, median frequency (MDF), and conduction velocity $(\mathrm{CV})$. The CV estimator had a higher $(p=0.002)$ rate of decrease at the end of the follicular phase and at the end of the luteal phase. The MDF $(p=0.002)$ and MNF ( $p=$ 0.004 ) estimators had a higher rate of decrease at the beginning of the follicular phase and at the end of the luteal phase. No significant differences between phases of the menstrual cycle were detected with the ARV and RMS estimators $(p>0.05)$. These results suggest that the behavior of the muscles in women presents different characteristics during different phases of the menstrual cycle. In particular, women were more susceptible to fatigue at the end of the luteal phase.
\end{abstract}

Keywords: electromyographic variables, menstrual cycle, muscular fatigue

(Some figures in this article are in colour only in the electronic version) 


\section{Introduction}

The study of injuries and muscle pain is relevant in areas such as ergonomics, sports and the treatment of chronic pain. Non-contact tears and rupture of the anterior cruciate ligament are the most common injuries in collective sports and athletics, and have received special attention by the scientific community (Sandler 2009, Farina and Merletti 2004). There is also special interest in shoulder and back pains (Rainoldi et al 2004, Salomoni 2008).

One consequence of muscle contraction is the increase in the concentration of lactic acid, a metabolic product (Cifrek et al 2009). During sustained isometric contractions, in which the muscle length and tension are held constant, the increased concentration of lactates is responsible for changes in intracellular $\mathrm{pH}$ and, as a result, muscle fiber conduction velocity (CV) decreases (Brody et al 1991), directly changing the shape of the motor unit action potential (MUAP) waveform (Cifrek et al 2009). This phenomenon is correlated with a decrease of the mean (MNF) and median (MDF) frequencies of the spectra of surface electromyographic (S-EMG) signals (Sadoyama et al 1988).

In several works, gender differences have been observed in muscular activities. Women are usually able to sustain a contraction for longer periods of time than men, especially at lower contraction intensities (Hicks et al 2001, Clark et al 2005, Hunter et al 2006), but not at maximal contractions (Baudry et al 2007). The most common explanations for these differences are men's greater muscle strength and women's lesser reliance on glycolytic metabolism. Men are typically stronger than women, thus they must activate a larger muscle mass to exert the same relative force, which will be accompanied by larger intramuscular pressures and a greater occlusion of blood flow (de Ruiter et al 2007). Muscle biopsies have shown lower glycolytic enzyme activity in women, indicating a decreased potential for anaerobic glycolysis (Ettinger 1998), which would enhance their endurance. In general, these differences lead to lower sensitivity to fatigue in women. This lower sensitivity may subject women's muscle fibers to long lasting fatigue and, in extreme cases, contribute to lesions (Gandevia 2001, Hägg et al 2004).

The menstrual cycle is another important factor influencing muscle fatigability in women. There is a strong link between hormone levels and laxity of joints, making women more vulnerable to injuries (Masterson 1999). The level of estrogen, which strengthens the muscles and tendons, is significantly reduced in the middle of the cycle. At the end of the cycle, the level of the relaxin hormone increases, which also weakens the tendons. The difference in concentration of sex hormones may also explain differences in neuromuscular control (Slauterbeck et al 2002). Women in menopause typically go through less intense hormonal changes, and generally display greater resistance to fatigue (Farina and Merletti 2004).

This work investigates the changes in several S-EMG estimators-root mean square (RMS) value, absolute rectified value (ARV), MNF, MDF and CV_-along four phases of the menstrual cycle: beginning and end of the follicular phase, and beginning and end of the luteal phase, respectively. S-EMG signals were collected during long-term isometric contractions performed in four weekly sessions, and the rates of change of estimator values during the contractions in each of the phases of the menstrual cycle were compared.

The aim of this work is to identify periods of higher and lower sensitivity to fatigue in women. This knowledge can potentially be used to optimize performance during physical training or rehabilitation therapy, for example. Also, this work may be of interest to those involved in the study of pathophysiology of chronic muscular pain, which is more frequent in women than in men, such as fibromyalgia. 


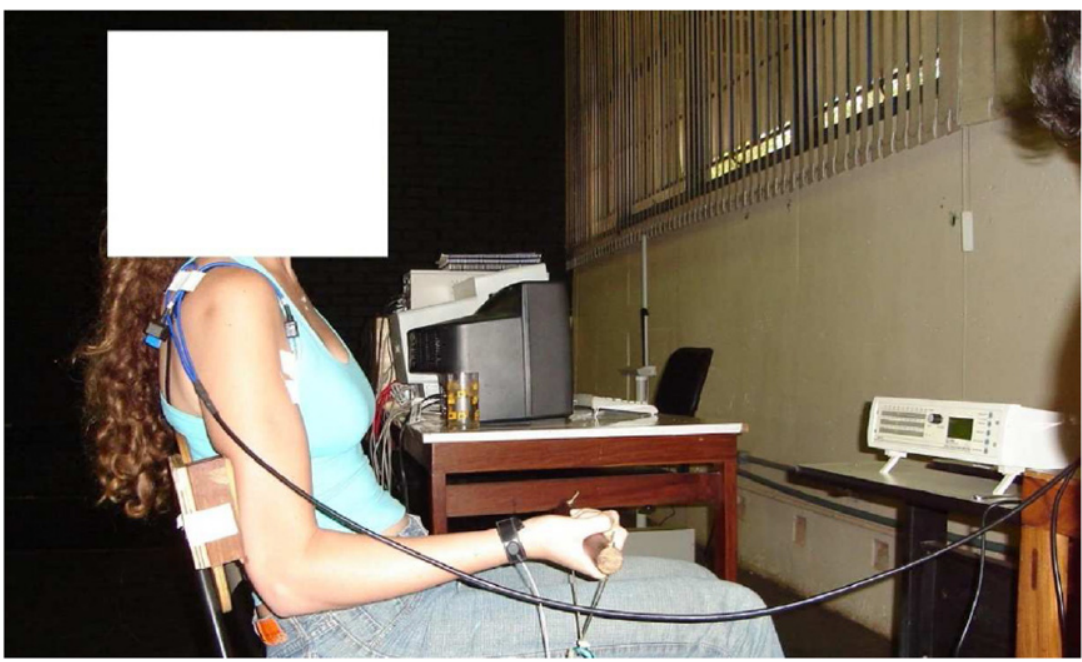

Figure 1. Position of the subject during the exercise. An adapted chair was used to fix the elbow such that the only possible movement of the arm was isometric elbow flexion. The load cell was fixed in a hook at the side of the chair with adjustable cables, such that the elbow joint was flexed at an angle of $90^{\circ}$.

\section{Methods}

\subsection{Subjects}

Twenty three female subjects volunteered to participate in the study. Due to desistance, hormonal problems and high noise levels in the S-EMG measurements, only ten volunteers $(24.0 \pm 2.8$ years of age) were included in the analysis. All subjects were right handed and had no known neurological disorders. All females had regular menstrual cycles, were not practicing regular exercises and were not using any hormonal medicine or contraceptive for at least 6 months. All subjects provided informed consent, and the experimental protocol was approved by the research ethics committee of the University of Brasilia.

\subsection{Experimental protocol}

The same experimental protocol was used with all subjects. Each subject performed the experimental protocol in four sessions, with an interval of 1 week between sessions.

The subjects sat on a chair specially adapted to firm the elbow such that the only possible movement of the arm was isometric elbow flexion (figure 1). This was done to minimize contractions of other muscles that could interfere with the results of the experiment.

An EMG 16 electromyograph (OT Bioelettronica s.n.c., Italy), connected to a laptop computer with a PCMCIA card, was used to acquire the S-EMG and force signals.

A load cell with $50 \mathrm{kgf}$ of maximal load, model TS (AEPH do Brasil, Sao Paulo, Brazil), connected to a biomechanical signal amplifier MISO II (OT Bioelettronica s.n.c., Italy), was used to measure the subjects' force level. The amplification gain was set to 1 . Three $3 \mathrm{~s}$ isometric contraction measurements were performed at the beginning of each session to determine the maximal voluntary contraction (MVC) of the subject. However, the MVC measured during the first session was adopted as MVC for all sessions; the other MVC 


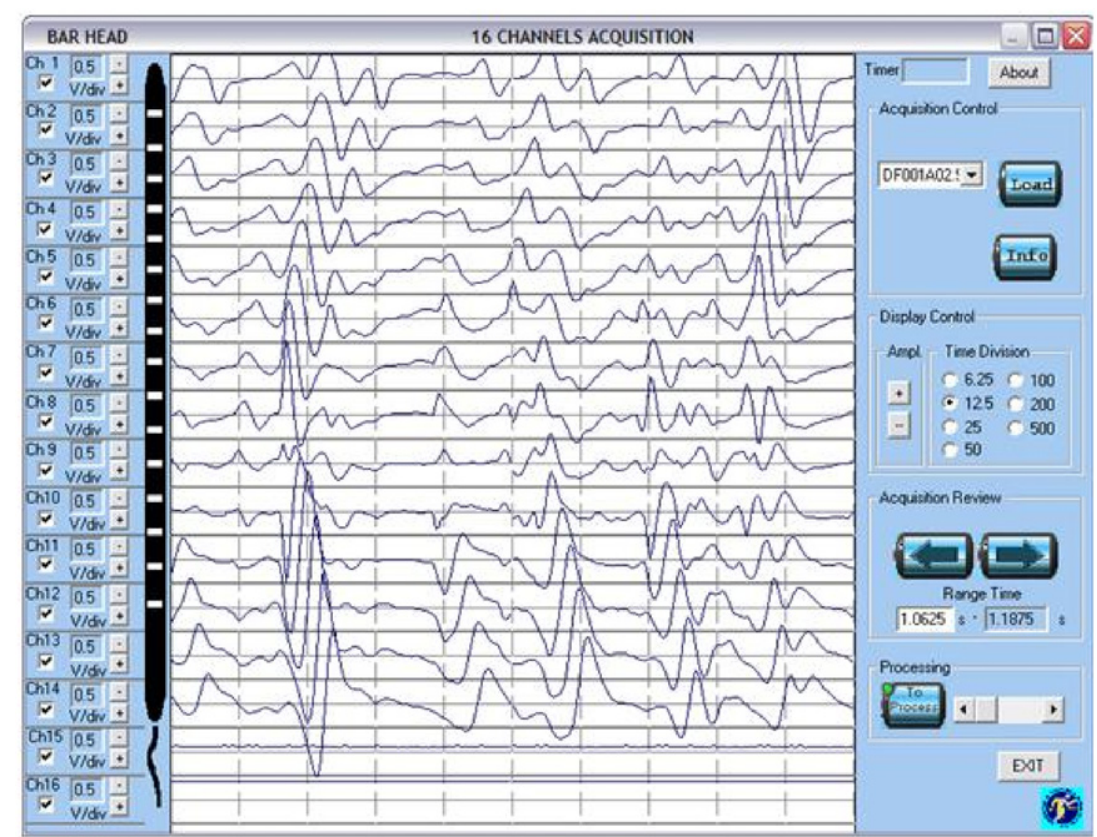

Figure 2. Mapping of the tendons and innervation zones of the biceps brachii muscle, using a 16electrode array (Ag, $10 \mathrm{~mm} \times 1 \mathrm{~mm}$ area, $5 \mathrm{~mm}$ inter-electrode distance, OT Bioelettronica s.n.c., Italy). In this example, an innervation zone was found near the pair of electrodes corresponding to channel 9. The optimal regions for S-EMG recording are the regions right above or right below this pair of electrodes.

acquisitions were performed only to guarantee the repeatability of the protocol in all sessions. Strong verbal encouragement was used for each MVC. After each MVC estimation, the subject rested for $1 \mathrm{~min}$.

A 16-electrode array $(\mathrm{Ag}, 10 \mathrm{~mm} \times 1 \mathrm{~mm}$ area, $5 \mathrm{~mm}$ inter-electrode distance, OT Bioelettronica s.n.c., Italy) was used for mapping the optimal S-EMG region of the biceps brachii in each session. The sample rate was $2048 \mathrm{~Hz}$ and an analog gain of 2000 in the single differential configuration was used. The force level was $30 \%$ of the MVC. A 3 s signal was used for the estimation of the optimal position. A sample result is shown in figure 2, where the innervation zone and optimal regions for acquisition are clearly distinguishable. After this effort, the subject rested for $2 \mathrm{~min}$.

After mapping, S-EMG signals were acquired with an array of eight surface electrodes $(\mathrm{Ag}-\mathrm{AgCl}, 10 \mathrm{~mm} \times 1 \mathrm{~mm}$ area, $5 \mathrm{~mm}$ inter-electrode distance, OT Bioelettronica s.n.c., Italy), which was placed on the optimal region of the short head of the biceps brachii. The skin was cleaned and conductive gel was applied between the skin and each electrode. The single differential configuration was used, resulting in one 7-channel S-EMG signal for each acquisition. A reference electrode was placed on the right wrist. A sample rate of $2048 \mathrm{~Hz}$ and an analog gain of 2000 were used. The position where the electrode array was placed is show in figure 3. After $5 \mathrm{~min}$ of rest, an isometric contraction was performed for $90 \mathrm{~s}$ using $40 \%$ of the previously registered MVC. A representative example of the S-EMG signals measured during this contraction is shown in figure 4 . 


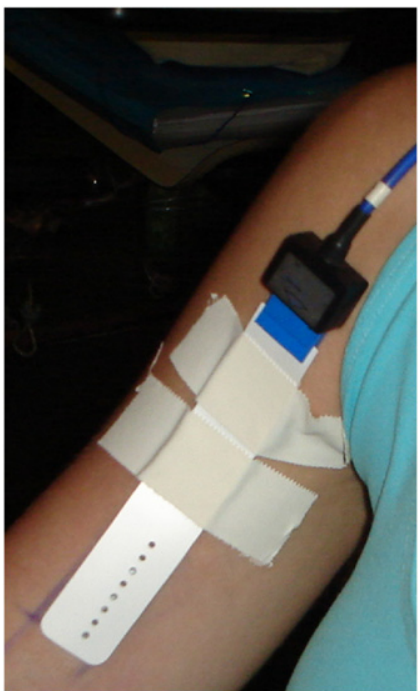

Figure 3. Placement of the flexible 8-electrode array $(\mathrm{Ag}-\mathrm{AgCl}, 10 \mathrm{~mm} \times 1 \mathrm{~mm}$ area, $5 \mathrm{~mm}$ inter-electrode distance, OT Bioelettronica s.n.c., Italy) on the optimal region of the short head of biceps brachii, after identification of the innervation zone. Conductive gel was applied between the skin and each electrode of the array.

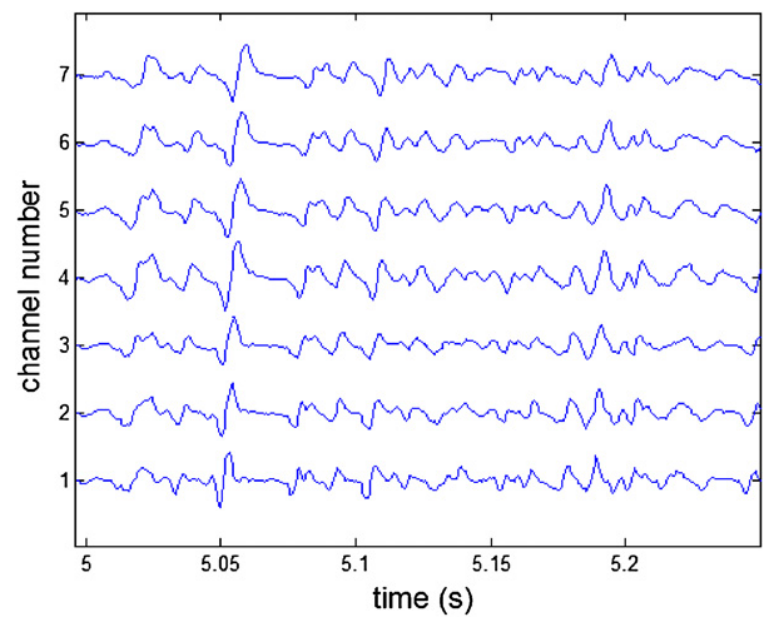

Figure 4. Segment of a 7-single differential S-EMG signal acquired with an 8-electrode array (Ag-AgCl, point electrode, $5 \mathrm{~mm}$ inter-electrode distance, OT Bioelettronica s.n.c., Italy) from the biceps brachii short head. The sampling frequency was $2048 \mathrm{~Hz}$. This segment has approximately $0.3 \mathrm{~s}$ of length.

\subsection{S-EMG estimators}

The signal was evaluated using five estimators: rms amplitude, average rectified value, MNF, MDF and CV. These estimators were calculated independently for each analysis window (a $\mathrm{N}$-sample segment of the signal), as described next. 
Signal amplitude. The most commonly used estimators for evaluating the amplitude characteristics of the S-EMG signal are the RMS amplitude and the average rectified value (Farina and Merletti 2000). These estimators were calculated for the channel with the highest signal amplitude (peak value) as follows:

$$
\begin{aligned}
& \mathrm{RMS}=\sqrt{\frac{1}{N} \sum_{n=1}^{N}|x[n]|^{2}} \\
& \mathrm{ARV}=\frac{1}{N} \sum_{n=1}^{N}|x[n]|,
\end{aligned}
$$

where $x[n]$ is the $n$-th sample of the S-EMG signal and $N$ is the total number of samples in the analysis window.

Spectral shift. The S-EMG signals' mean and median frequencies were calculated, in order to estimate the signals' spectral changes as a function of time, due to fatigue. These two estimators are often used in combination (Farina and Merletti 2000), because each one has advantages and disadvantages over the other. MDF values are less susceptible to noise and more sensitive to fatigue than MNF values (Farina and Merletti 2000, Stulen and de Luca 1981). On the other hand, MNF values typically present smaller standard deviation than MDF values (Farina and Merletti 2000, Stulen and de Luca 1981). These estimators were calculated for the channel with the highest signal amplitude (peak value) as follows:

$$
\begin{aligned}
\mathrm{MNF} & =\frac{\sum_{m=1}^{M} f_{m} P[m]}{\sum_{m=1}^{M} P[m]}, \\
\mathrm{MDF} & =f_{m_{o}} \text { s.t. } \sum_{m=1}^{m_{o}} P[m] \approx \sum_{m=m_{o}}^{M} P[m] \approx \frac{1}{2} \sum_{m=1}^{M} P[m],
\end{aligned}
$$

where $P[m]$ is the $m$-th frequency bin of the power spectral density function of $x[n]$, and $f_{m}$ is the frequency (in Hz) associated with the $m$-th bin (Farina and Merletti 2000). The power spectra were calculated by taking the squared magnitude of the discrete Fourier transform of the signals. Only positive frequencies were used, i.e. $f_{0}=0 \mathrm{~Hz}$ and $f_{M+1}=f_{s} / 2$, where $f_{s}$ is the sampling frequency.

Conduction Velocity. We used the algorithm proposed by Farina et al (2001) for calculating CV from S-EMG signals acquired with an array of electrodes, with adaptations. This algorithm uses maximum likelihood estimation (MLE) and a generalization of the classical spectral waveform alignment algorithm (McGill and Dorfman 1984). The algorithm is based on the idea that by assuming the $\mathrm{CV}$ to be constant during voluntary isometric contractions of low intensity, and by knowing the inter-electrode distance, the estimation of the delay between adjacent channels allows the direct calculation of the mean CV. The problem is moved to the frequency domain in order to improve accuracy. The CV is estimated with high precision by finding the temporal shift (or frequency-domain phase) that minimizes the mean squared difference between the spectra of adjacent channels. Our implementation of the algorithm follows the following steps.

We used a single differential low-pass filter to highlight the peaks of the signal, allowing for a more accurate result (McGill and Dorfman 1984). The filter's difference equation is

$$
y[n]=x[n+1]-x[n-1],
$$


where $x[n]$ is the $n$-th sample of the original signal and $y[n]$ is the $n$-th sample of the filtered signal. The above filter also reduces high-frequency noise, as opposed to a true differentiator, which enhances high-frequency components (McGill and Dorfman 1984).

The multi-channel S-EMG signal may be modeled as follows. In the ideal case, the signals detected by different electrodes along the muscle fibers are merely delayed versions of each other, plus white Gaussian noise:

$$
y_{k}[n]=s[n-(k-1) \theta]+w[n],
$$

where $s[n]$ is the $n$-th sample of the basic waveform, $k=1, \ldots, K$ is the channel number, $\theta$ is associated with the temporal delay between adjacent channels-the actual inter-electrode temporal delay is given by $\left(\theta \times f_{s}\right)^{-1}$ - and $w[n]$ is white Gaussian noise.

The basic waveform was estimated by aligning and averaging the signals that were measured in each of the $K$ channels (Farina et al 2001) as follows:

$$
\hat{s}[n]=\frac{1}{K} \sum_{k=1}^{K} y_{k}[n+(k-1) \theta] .
$$

Assuming a constant $\mathrm{CV}$ in the region of measurement, the estimation of $\theta$ allows the direct calculation of the CV. The maximum likelihood estimate is the value of the delay that minimizes the mean squared error of all signals with respect to the estimated basic signal, $\hat{s}[n]$. The total squared error is

$$
e_{\text {MLE }}^{2}=\sum_{k=1}^{K} \sum_{n=1}^{N}\left(y_{k}[n]-\hat{s}[n-(k-1) \theta]\right)^{2},
$$

where $N$ is the total number of samples in the analysis window. This may be rewritten (Farina et al 2001) as

$$
\begin{aligned}
& e_{\mathrm{MLE}}^{2}=\left(1-\frac{1}{K}\right) \sum_{k=1}^{K} e_{k}^{2} \\
& e_{k}^{2}=\sum_{n=1}^{N}\left(y_{k}[n]-\frac{1}{K-1} \sum_{i=1, i \neq k}^{K} y_{i}[n+(i-k) \theta]\right)^{2} .
\end{aligned}
$$

If $e_{\mathrm{MLE}}^{2}$ is minimized in the time domain, the accuracy in $\theta$ estimation will be limited by the sampling frequency. In order to avoid this limitation, the equation was carried to the frequency domain (McGill and Dorfman 1984, Farina et al 2001), in which $\theta$ is no longer a discrete-valued (quantized) variable. Equation (10) becomes

$$
e_{k}^{2}=\frac{2}{N} \sum_{m=1}^{N / 2}\left|Y_{k}[m]-\frac{1}{K-1} \sum_{i=1, i \neq k}^{K} Y_{i}[m] \mathrm{e}^{\frac{j 2 \pi m(i-k) \theta}{N}}\right|^{2},
$$

where $Y_{i}$ and $Y_{k}$ are the discrete Fourier transforms of $y_{i}$ and $y_{k}$, respectively, calculated as follows: $Y_{k}[m]=\sum_{n=1}^{N} y_{k}[n] \mathrm{e}^{-j 2 \pi(m-1)(n-1) / N}$.

The value of $\theta$ that minimizes $e_{\text {MLE }}^{2}$ was calculated using the Newton-Raphson method. We used an initial value of 0.39 for $\theta$. This corresponds to a $\mathrm{CV}$ of $4 \mathrm{~m} \mathrm{~s}^{-1}$, which is within the range of typical physiological values $\left(2-7 \mathrm{~m} \mathrm{~s}^{-1}\right.$ (Farina et al 2001)). The stop criteria were (i) $e_{\text {MLE }}^{2}<5 \times 10^{-5}$, or (ii) a maximum of 25 interactions. All the signals analyzed in this work have met the first criterion. However, it is important to include a second criterion in order to avoid an infinite loop in the algorithm. 
Once the optimal value of $\theta$ had been estimated, CV was calculated as follows:

$$
\mathrm{CV}=d \times \theta \times f_{s},
$$

where $d$ is the inter-electrode distance.

We used data from only three adjacent electrodes (two differential channels) for CV estimation. This is because we observed that, in many of the exercise sessions, one or more channels did not present acceptable signal quality, due to poor electrode contact with the skin. When using an 8-electrode array, a single electrode with poor contact may render up to two differential channels to be useless. As a result, out of the seven differential channels, only two or three adjacent channels would provide acceptable signal. Thus, in order to avoid discarding subjects, we used only two channels for CV estimation $(K=2)$. For each exercise session, two channels were selected by estimating the cross-correlation between each pair of differential channels, and then selecting the pair with the highest cross-correlation coefficient. In all selected pairs, the cross-correlation was greater than $75 \%$.

\subsection{Fatigue quantitation}

The S-EMG estimators described above (RMS, ARV, MDF, MNF and CV) were calculated independently for multiple segments of each signal. A 3.6 s rectangular sliding window was used for $\mathrm{CV}$, with a window step of $1.8 \mathrm{~s}$. A $0.5 \mathrm{~s}$ rectangular sliding window was used for the other four estimators, with a window step of $0.25 \mathrm{~s}$. The window was longer for CV because the calculation of this estimator is more computationally demanding.

The above process results in a temporal function associated with each estimator. The rate in which each estimator varies during the acquisition was estimated by linear regression. The estimator values and the angular coefficient of the regression were normalized by dividing by the linear coefficient of the regression's linear equation. This normalization was performed to compensate for biases due to differences in subcutaneous tissue thickness. The normalized angular coefficients were used for evaluating fatigue.

\subsection{Analysis tool}

An analysis tool was designed in Matlab (The MathWorks, Inc., Natick, MA, USA) to calculate the S-EMG estimators evaluated in this work: RMS, ARV, MNF, MDF and CV.

On the tool's setup window (figure 5), the user enters the following information: the name of the file that contains the S-EMG data, the length of the analysis window, the acquisition channels to be processed, the graphs to be displayed, the inter-electrode distance, the sampling rate, the MVC value and percentage and the name of the file in which the results are to be saved. When the 'ok' button is pressed, the selected graphs are displayed and the calculated estimators for each analysis window are saved onto a Microsoft Excel 2007-compatible spreadsheet file.

All S-EMG estimators were calculated according to equations (1)-(4) and (12). The Matlab tool allows the selection of a subset of differential channels, so that the user can discard channels associated with innervation zones or tendon regions.

\subsection{Data analysis}

The date of each subject's first session was scheduled without considering the subject's menstrual cycle, i.e. some of the subjects began the protocol during the follicular phase, others during the luteal phase (table 1). After 4 weeks, the results from each session of each subject were cyclically reordered so that the first set of results would correspond to the session that 


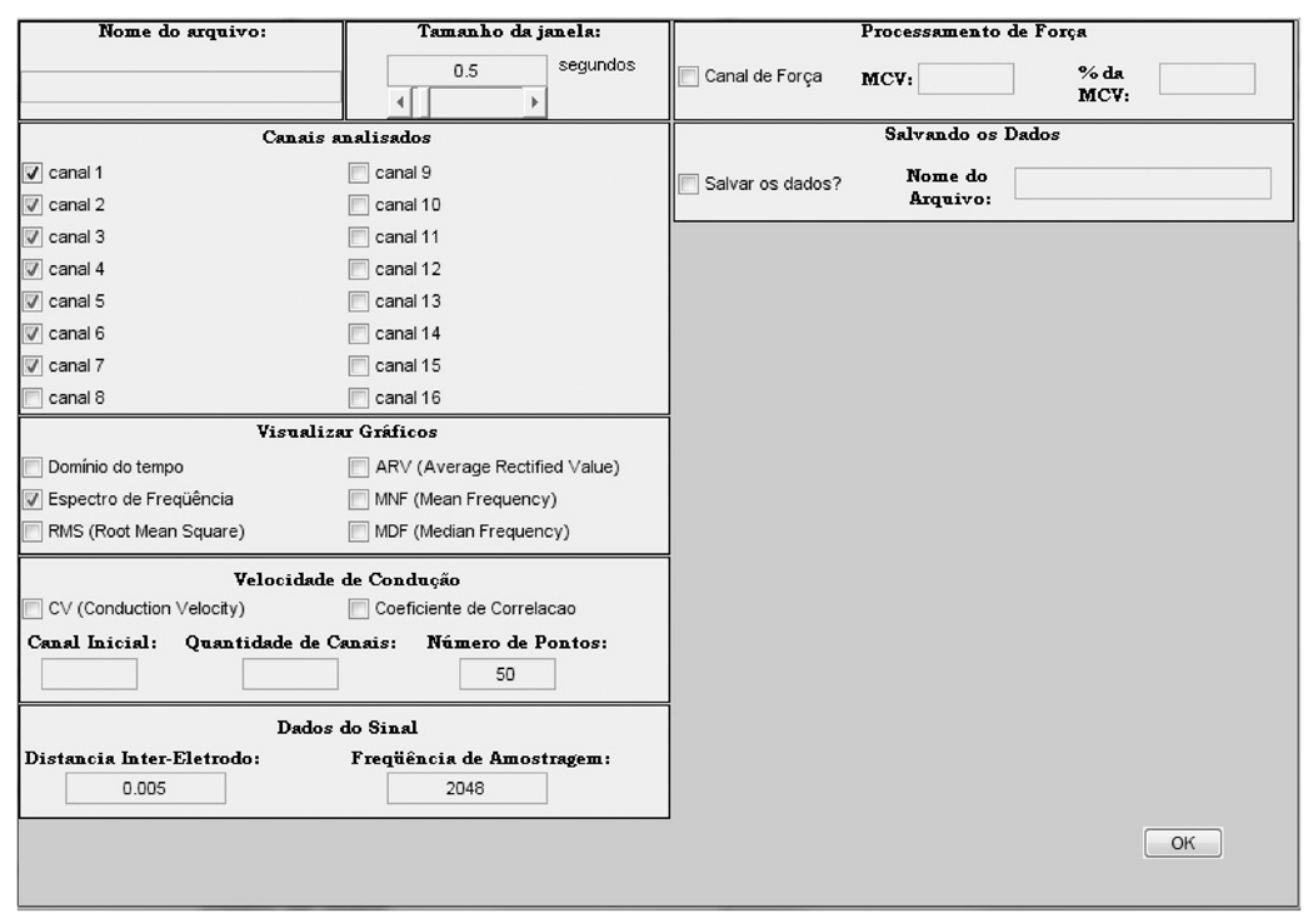

Figure 5. S-EMG signal analysis tool: setup window. After starting the program on Matlab, this window is shown and the following information is entered by the user: name of the input file, number of channels to be analyzed, number of channels for CV estimation, starting channel for CV estimation, size of the window used for processing, graphs to be displayed, sampling frequency, inter-electrode distance, MVC and MVC percentage level, and name of the output file.

Table 1. Cyclic session labeling, based on the week during which menses occurred.

\begin{tabular}{lllll}
\hline Week of menses & Session 1 & Session 2 & Session 3 & Session 4 \\
\hline First & F1 & F2 & L1 & L2 \\
Second & L2 & F1 & F2 & L1 \\
Third & L1 & L2 & F1 & F2 \\
Fourth & F2 & L1 & L2 & F1 \\
\hline
\end{tabular}

took place on the date closest to the subject's menses. After reordering, the results from each session were labeled F1 and F2 for the follicular phase and L1 and L2 for the luteal phase. For example, if the second session took place one day after the subject's menses, then the second session was considered as the first week of the menstrual cycle and was labeled F1, the third session was considered as the second week and was labeled F2, the fourth session was considered as the third week and was labeled L1 and the first session was considered as the fourth week and was labeled L2.

The analysis tool shown in figure 5 was used for calculating the fatigue estimators from the acquired S-EMG signals. 


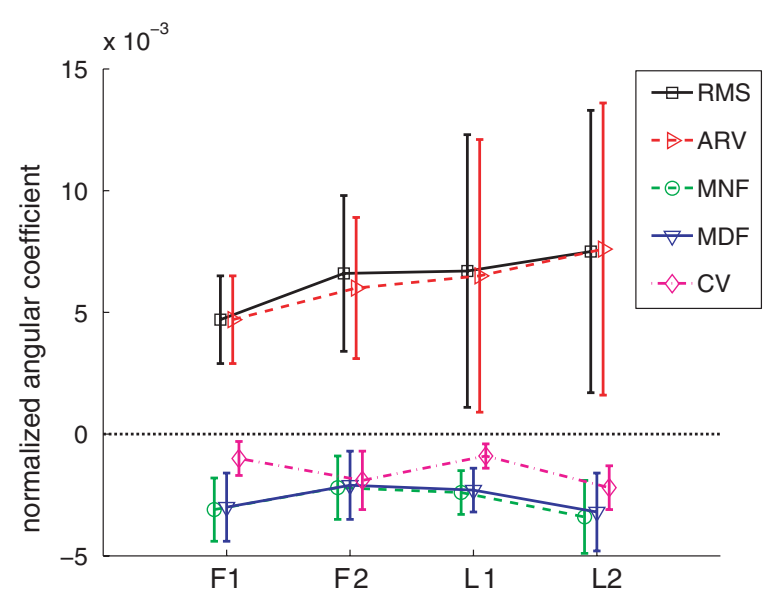

Figure 6. Normalized regression angular coefficients (mean and standard deviation) for different S-EMG estimators (RMS, ARV, MNF, MDF and CV) for each phase of the menstrual cycle (F1, F2, $\mathrm{L} 1$, and L2). The data have passed the Shapiro-Wilk normality test $(p>0.05)$. The differences in the rate of decrease in CV between different phases of the menstrual cycle were statistically significant. Subjects presented a significantly higher rate of decrease in CV during phases F2 and L2 than during phases F1 and L1. Significant differences were also found for the angular coefficients calculated from the MNF and MDF variables. A significantly higher rate of decrease in MNF and MDF was observed during phases F1 and L2 than during phases F2 and L1. The differences were not significant for the angular coefficients calculated from the ARV and RMS variables.

\subsection{Statistical analysis}

The normalized value of the angular coefficient calculated for each estimator (RMS, ARV, MNF, MDF and CV) and for each phase of the menstrual cycle (F1, F2, L1 and L2) was tested by Shapiro-Wilk's normality test. In the Shapiro-Wilk test, the null hypothesis is that the population is normally distributed. If the $p$-value is less than the chosen alpha level, then the null hypothesis is rejected (i.e. the data are not from a normally-distributed population). If the $p$-value is greater than the chosen alpha level, then the null hypothesis is not rejected. For example, for an alpha level of 0.05 , a data set with a $p$-value of 0.40 does not result in rejection of the hypothesis that the data are from a normally distributed population. Bartlett's test was used to evaluate the homoscedasticity of the distribution. ANOVA for repeated measures was performed to evaluate if there was significant difference between the data from different phases of the menstrual cycle. Bonferroni's post hoc test was then used to identify which estimators and which phases of the menstrual cycle presented significant differences in the rate of increase or decrease.

\section{Results}

The normalized angular coefficients for each estimator in each phase of the menstrual cycle for all selected subjects are shown in table 2 .

Figure 6 shows the mean normalized angular coefficients (and standard deviations) for each estimator in each phase of the menstrual cycle. The normalized angular coefficients of all the estimators follow a normal distribution, according to the Shapiro-Wilk normality test $(p>0.05)$. 
Table 2. Normalized angular coefficients for each estimator in each phase of the menstrual cycle, for all analyzed subjects. F1, F2, L1 and L2 stand for follicular 1, follicular 2, luteal 1 and luteal 2 , respectively. $\mathrm{P}$ stands for phase, $\mu$ stands for mean and $\sigma$ stands for standard deviation. The normalized angular coefficients represent the angular coefficient, $a$, in the regression's normalized linear equation $y(x)=a x+1$. All values were multiplied by 1000 for visualization purposes.

\begin{tabular}{|c|c|c|c|c|c|c|c|c|c|c|c|}
\hline \multirow[b]{2}{*}{$\mathrm{P}$} & \multicolumn{10}{|c|}{ Subjects } & \multirow[b]{2}{*}{$\mu \pm \sigma$} \\
\hline & 1 & 2 & 3 & 4 & 5 & 6 & 7 & 8 & 9 & 10 & \\
\hline \multicolumn{12}{|c|}{ RMS (normalized angular coefficients $\times 1000$ ) } \\
\hline F1 & 3.2 & 2.3 & 3.9 & 7.1 & 5.5 & 2.7 & 7.0 & 5.5 & 6.7 & 3.1 & $4.7 \pm 1.8$ \\
\hline $\mathrm{F} 2$ & 3.8 & 6.0 & 7.8 & 10.1 & 3.4 & 3.6 & 11.4 & 11.6 & 3.1 & 5.3 & $6.6 \pm 3.2$ \\
\hline L1 & -3.7 & 7.7 & 7.9 & 3.3 & 5.8 & 7.4 & 17.9 & 7.0 & 12.4 & 1.0 & $6.7 \pm 5.6$ \\
\hline L2 & -1.8 & 4.7 & 6.4 & 6.7 & 14.8 & 0.3 & 18.3 & 11.7 & 7.1 & 6.7 & $7.5 \pm 5.8$ \\
\hline
\end{tabular}

ARV (normalized angular coefficients $\times 1000$ )

\begin{tabular}{llllllllllll}
\hline F1 & 2.6 & 2.5 & 3.7 & 7.6 & 5.4 & 2.8 & 6.3 & 5.5 & 6.9 & 3.2 & $4.7 \pm 1.8$ \\
F2 & 3.1 & 6.5 & 7.7 & 5.1 & 3.7 & 3.8 & 11.2 & 1.07 & 2.7 & 5.1 & $6.0 \pm 2.9$ \\
L1 & -4.1 & 7.5 & 7.8 & 3.2 & 5.7 & 7.3 & 17.6 & 6.6 & 12.1 & 0.9 & $6.5 \pm 5.6$ \\
L2 & -2.3 & 5.0 & 6.4 & 6.9 & 14.8 & 0.5 & 18.4 & 12.4 & 6.8 & 7.0 & $7.6 \pm 6.0$ \\
\hline
\end{tabular}

MNF (normalized angular coefficients $\times 1000)$

\begin{tabular}{llllllllllll}
\hline F1 & -1.1 & -3.2 & -2.1 & -6.5 & -3.1 & -3.5 & -2.9 & -2.7 & -3.5 & -2.0 & $-3.1 \pm 1.3$ \\
F2 & -0.4 & -3.9 & -2.0 & -5.3 & -2.1 & -2.0 & -2.1 & -1.4 & -1.3 & -1.7 & $-2.2 \pm 1.3$ \\
L1 & -0.5 & -2.6 & -3.0 & -3.4 & -2.7 & -2.9 & -3.0 & -2.0 & -2.6 & -1.2 & $-2.4 \pm 0.9$ \\
L2 & -0.3 & -3.8 & -2.7 & -5.8 & -4.3 & -3.1 & -5.6 & -3.4 & -2.2 & -2.7 & $-3.4 \pm 1.5$ \\
\hline
\end{tabular}

MDF (normalized angular coefficients $\times 1000$ )

\begin{tabular}{llllllllllll}
\hline F1 & -1.4 & -3.4 & -2.1 & -6.2 & -3.0 & -4.1 & -2.5 & -2.8 & -3.6 & -1.3 & $-3.0 \pm 1.4$ \\
F2 & 0.1 & -3.7 & -1.7 & -5.2 & -2.0 & -2.3 & -2.1 & -1.4 & -1.0 & -1.4 & $-2.1 \pm 1.4$ \\
L1 & -0.1 & -2.8 & -2.7 & -3.4 & -2.6 & -3.0 & -2.8 & -1.9 & -2.1 & -1.3 & $-2.3 \pm 0.9$ \\
L2 & -0.3 & -4.0 & -2.6 & -5.9 & -4.0 & -2.8 & -5.4 & -3.2 & -1.8 & -2.5 & $-3.2 \pm 1.6$ \\
\hline
\end{tabular}

$\mathrm{CV}$ (Normalized angular coefficients $\times 1000)$

\begin{tabular}{llllllllllll}
\hline F1 & -1.2 & -1.7 & 0.03 & -0.8 & -0.2 & -1.4 & -0.4 & -1.7 & -2.0 & -0.8 & $-1.0 \pm 0.7$ \\
F2 & -1.0 & -4.0 & -1.1 & -3.7 & -1.9 & -1.2 & -3.1 & -0.5 & -1.4 & -1.3 & $-1.9 \pm 1.2$ \\
L1 & -0.1 & -0.5 & -0.1 & -1.8 & -1.0 & -0.8 & -1.0 & -0.9 & -0.6 & -1.7 & $-0.9 \pm 0.5$ \\
L2 & -0.4 & -1.4 & -1.5 & -3.8 & -3.0 & -2.3 & -3.1 & -1.9 & -2.5 & -2.1 & $-2.2 \pm 0.9$ \\
\hline
\end{tabular}

According to the ANOVA test, significant difference was found in the CV $(p=0.002)$, $\operatorname{MDF}(p=0.028)$ and MNF $(p=0.004)$ angular coefficient values.

For the CV estimator, phases F2 and L2 had higher mean slopes $(-0.0019$ and -0.0022$)$, and phases F1 and L1 had lower mean slopes $(-0.0010$ and -0.0009$)$. Bonferroni's test indicated statistically significant differences between groups F1 and L2 ( $p=0.022)$, F2 and L1 $(p=0.045)$ and L1 and L2 $(p=0.007)$. Even though there seems to be a difference between groups F1 and F2, this was not statistically significant $(p=0.119)$ for this number of subjects $(n=10)$. No significant differences were found between groups F1 and L1 and between groups F2 and L2.

The MDF $(p=0.002)$ and MNF ( $p=0.004)$ estimators had a higher rate of decrease during phases F1 and L2. For the MDF estimator, significant differences were found between 


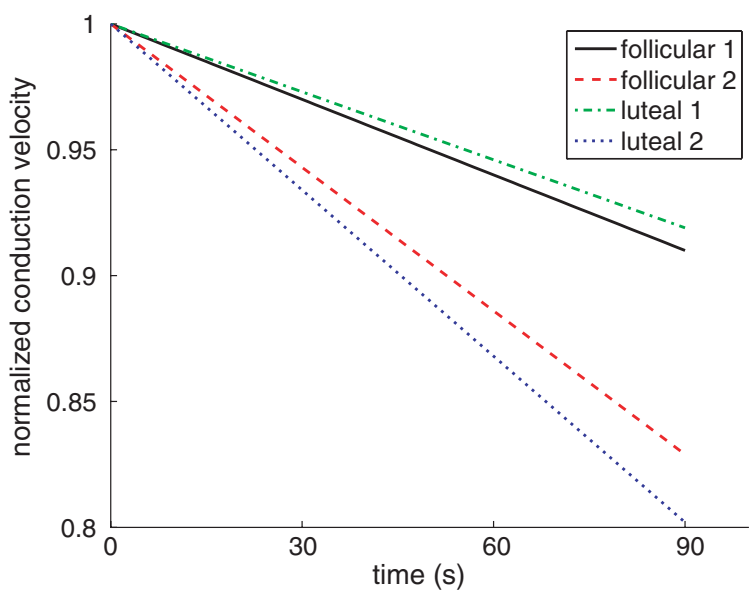

Figure 7. Mean normalized regression lines obtained with the CV estimator for each phase of the menstrual cycle, during the 90 s contractions. Phases F2 and L2 presented a higher rate of decrease in $\mathrm{CV}$ than phases F1 and L1, which suggests that the volunteers were more susceptible to fatigue during those periods.

groups F1 and F2 $(p=0.043), \mathrm{F} 2$ and L2 $(p=0.009)$ and L1 and L2 $(p=0.037)$. For the MNF estimator, greater differences were found between groups F1 and F2 $(p=0.010)$, and $\mathrm{L} 1$ and $\mathrm{L} 2(p=0.036)$.

The ANOVA test found no significant differences in RMS $(p=0.35)$ and ARV values $(p=0.31)$. However, there was a clear increase in the variance of the angular coefficients from phase F1 to phase L2, for both estimators (figure 6).

Figure 7 presents the mean normalized regression lines obtained with the CV estimator for each phase of the menstrual cycle during the $90 \mathrm{~s}$ contractions. Note that phases F2 and L2 present faster decreases in CV than F1 and L1, which suggests that the volunteers were more susceptible to fatigue during those periods.

\section{Discussion}

The main goal of this work was to study fatigue in women trough the menstrual cycle. Shifts in the S-EMG frequency spectrum are the most traditional fatigue indicators in the literature. The results observed with the frequency-domain estimators (MNF and MDF) showed higher rate of decrease during phases F1 and L2 than during phases F2 and L1 (figure 6). Most of these differences were statistically significant.

Decreases in $\mathrm{CV}$ might represent a more robust and reliable fatigue indicator than decreases in MNF and MDF, because CV provides information not only about changes in the frequency spectrum (De Luca 1984) but also regarding fiber membrane properties and peripheral muscle fatigue (Farina 2002). CV changes are also indicative of muscle fibertype constituency and particular muscle training (Sadoyama et al 1988). Based on the CV estimator, phases F1 and L1 presented a lower level of fatigue (slower decrease of CV over time), while the fatigability during phases F2 and L2 was higher. The differences between the results associated with the CV estimator and with the MNF and MDF estimators may be due to factors such as differences in fiber recruitment strategies. 
The increased fatigability in females during certain periods of the menstrual cycle indicates a need for special attention. According to the overload injury model (Kibler et al 1992), exercise with fatigue and repetition is related to musculo-tendinous injuries. Repetitive muscle use may eventually lead to inflexibility, muscle weakness and imbalance in muscle strength in a particular area. Continued use may cause pathological injury. Overuse injuries are the most common type of injury in exercising populations.

The phases of the menstrual cycle in which higher fatigability was observed with the $\mathrm{CV}$ estimator (F2 and L2) coincide with periods of sharp estrogen decreases (Hewett et al 2006). Estrogen has direct effects on muscle, and fluctuations in levels of estrogen and of other female sex hormones may play a role in dynamic muscle control (Hewett et al 2007). Estrogen receptors have been reported in skeletal muscle (Huijing and Jaspers 2005). Recent reports that estrogen does not influence the mechanical properties of knee ligaments suggest that the effects of the menstrual cycle and hormones may be related to neuromuscular effects (Warden et al 2006). In addition, it has been shown that fluctuations in estrogen levels may affect neuromuscular firing patterns in female athletes (Florini 1986). These findings are corroborated by a study that showed that athletes taking hormonal contraceptives had a lower injury rate, because the elevated estrogen and progesterone levels in oral contraceptive pills inhibit ovulatory hormone oscillations and prevent ovulation directed by the hypothalamus (Moller-Nielson and Hammar 1991).

No significant differences during the menstrual cycle were observed with the time-domain amplitude estimators (RMS and ARV). S-EMG signal amplitude depends not only on the fatigue state, but also on the force production of the muscle under test. Changes in signal amplitude cannot be unequivocally attributed to muscular fatigue (Luttmann et al 2000). Although these amplitude-based estimators were unable to provide significant indication of increased fatigue, a progressive increase in the variance of these estimators was observed through the menstrual cycle (see figure 6).

\section{Conclusion}

The results obtained with the $\mathrm{CV}$ estimator suggest that females are more susceptible to fatigue during the end of both follicular and luteal phases of the menstrual cycle. These phases coincide with periods of high decrease in hormone concentration. Frequency-domain estimators indicated increased fatigue on different phases of the menstrual cycle. These differences could be due to factors such as fiber recruitment strategies or firing rate differences, for example. However, both results suggest a significant variation along the menstrual cycle in susceptibility to muscular fatigue during isometric contractions.

Fatigue levels may be associated with injuries (Gefen 2002, Stacoff et al 1996, Grimston et al 1993). Therefore, ignoring the hormonal fluctuations in females on training programs or in sports may increase the risk of muscular injuries. The results herein presented suggest that individual training programs that take into account the menstrual cycle may lead to better performance by female athletes.

\section{References}

Baudry S, Klass M, Pasquet B and Duchateau J 2007 Age-related fatigability of the ankle dorsiflexor muscles during concentric and eccentric contractions Eur. J. Appl. Physiol. 100 515-25

Brody L R, Pollock M T, Roy S H, De Luca C J and Celli B 1991 pH-induced effects on median frequency and conduction velocity of the myoelectric signal J. Appl. Physiol. 71 1878-85 
Cifrek M, Medved V, Tonković S and Ostojić S 2009 Surface EMG-based muscle fatigue evaluation in biomechanics Clin. Biomech. 24 327-40

Clark B C, Collier S R, Manini T M and Ploutz-Snyder L L 2005 Sex differences in muscle fatigability and activation patterns of the human quadriceps femoris Eur. J. Appl. Physiol. 94 196-206

De Luca C J 1984 Myoelectrical manifestations of localized muscular fatigue in humans Crit. Rev. Biomed. Eng. 11 251-79

de Ruiter C J et al 2007 The isometric torque at which knee-extensor muscle reoxygenation stops Med. Sci. Sports Exerc. 39 443-53

Ettinger S M 1998 Muscle sympathetic nerve activity during exercise and the influences of gender Gender Differences in Metabolism: Practical and Nutritional Implications ed M Tarnopolsky (Boca Raton, FL: CRC Press) pp 87119

Farina D, Arendt-Nielsen L, Merletti R and Graven-Nielsen T 2002 Assessment of single motor unit conduction velocity during sustained contractions of the tibialis anterior muscle with advanced spike triggered averaging J. Neurosci. Methods 115 1-12

Farina D and Merletti R 2000 Comparison of algorithms for estimation of EMG variables during voluntary isometric contractions J. Electromyogr. Kinesol. $10337-49$

Farina D and Merletti R 2004 Methods for estimating muscle fiber conduction velocity from surface electromyographic signals Med. Biol. Eng. Comput. 42 432-45

Farina D, Muhammad W, Fortunato E, Mestre O, Merletti R and Rix H 2001 Estimation of single motor unit conduction velocity from surface electromyogram signals detected with linear electrode arrays Med. Biol. Eng. Comput. 39 225-36

Florini J R 1986 Hormonal control of muscle growth Muscle Nerve 10 577-98

Gandevia S C 2001 Spinal and supraspinal factors in human muscle fatigue Physiol. Rev. 81 1725-89

Gefen A 2002 Biomechanical analysis of fatigue-related foot injury mechanisms in athletes and recruits during intensive marching Med. Biol. Eng. Comput. 40 302-10

Grimston S K and Zernicke R F 1993 Exercise-related stress response in bone. J. Appl. Biomech. 9 2-14

Hägg G M, Melin B and Kadefors R 2004 Applications in ergonomics Electromyography: Physiology, Engineering, and Non-invasive Applications ed R Merletti and P A Parker (Hoboken, NJ: Wiley) pp 343-63

Hewett T E, Myer G D and Ford K R 2006 Anterior cruciate ligament injuries in female athletes: part 1. Mechanisms and risk factors. Am. J. Sports Med. 34 299-311

Hewett T E, Zazulak B T and Myer G D 2007 Effects of the menstrual cycle on anterior cruciate ligament injury risk: a systematic review Am. J. Sports Med. 35 659-68

Hicks A L, Kent-Braun J and Ditor D S 2001 Sex differences in human skeletal muscle fatigue Exerc. Sports Sci. Rev. 29 109-12

Huijing P A and Jaspers R T 2005 Adaptation of muscle size and myofascial force transmission: a review of some new experimental results Scand. J. Med. Sci. Sports 15 349-80

Hunter S K, Butler J E, Todd G, Gandevia S C and Taylor J L 2006 Supraspinal fatigue does not explain the sex difference in muscle fatigue of maximal contractions J. Appl. Physiol. 101 1036-44

Kibler W B, Chandler T J and Stracener E S 1992 Musculoskeletal adaptations and injuries due to overtraining. Exerc. Sports Sci. Rev. 20 99-126

Luttmann A, Jager M and Laurig W 2000 Electromyographical indication of muscular fatigue in occupational field studies Int. J. Ind. Ergon. 25 645-60

Masterson G 1999 The impact of menstrual phases on anaerobic power performance in collegiate women J. Strength Cond. Res. 13 325-9

McGill K C and Dorfman L J 1984 High-resolution alignment of sampled waveforms IEEE Trans. Biomed. Eng. 31 462-8

Moller-Nielson J and Hammar M 1991 Sports injuries and oral contraceptive use. Is there a relationship? Sports Med. 12 152-60

Rainoldi A, Casale R, Hodges P and Jull G 2004 Applications in rehabilitation medicine and related fields Electromyography: Physiology, Engineering, and Non-invasive Applications ed R Merletti and P A Parker (Hoboken, NJ: Wiley) pp 403-33

Sadoyama T, Masuda T, Miyata H and Katsuta S 1988 Fiber conduction velocity and fiber composition in human vastus lateralis Eur. J. Appl. Physiol. 57 767-71

Salomoni S 2008 Effect of gender differences and the female menstrual cycle on fatigue and its impact on surface electromiographic variables MSc Dissertation University of Brasilia, Brazil (in Portuguese)

Sandler S 2009 The Association Between Joint Laxity and Female Hormones: Changes in Joint Laxity Associated with the Menstrual Cycle, with Pregnancy, with the Post-Partum Period, and with the Menopause (Saarbrücken: VDM Verlag) 
Slauterbeck J R, Fuzie S F, Smith M P, Clark R J, Tom Xu K, Starch D W and Hardy D M 2002 The menstrual cycle, sex hormones and anterior cruciate ligament injury J. Athletic Train. 37 275-80

Stacoff A, Steger S, Stussi E and Reinschmidt C 1996 Lateral stability in the sideward cutting movements Med. Sci. Sport Exerc. 28 350-8

Stulen F B and de Luca C J 1981 Frequency parameters of the myoelectric signals as a mensure of muscle conduction velocity IEEE Trans. Biomed. Eng. 28 515-23

Warden S J, Saxon L K, Castillo A B and Turner C H 2006 Knee ligament mechanical properties are not influenced by estrogen or its receptors. Am. J. Physiol. Endocrinol. Metab. 290 1034-40 